\title{
Determination of the Necessary Number of Technicians on the Faculty
}

\author{
Tomislav Šuh ${ }^{1}$, Dragan Mitić ${ }^{2}$ Dragica Lebl-Antonić ${ }^{3}$, \\ Aleksandar Lebl ${ }^{4}$ \\ ${ }^{1}$ Institute for Telecommunications and Electronics, IRITEL A.D. BELGRADE \\ Batajnički put 23, 11080 Belgrade, Serbia; E-mail: suh@iritel.com \\ ${ }^{2}$ Institute for Telecommunications and Electronics, IRITEL A.D. BELGRADE \\ Batajnički put 23, 11080 Belgrade, Serbia; E-mail: mita@iritel.com \\ ${ }^{3}$ Faculty of Pharmacy, Vojvode Stepe 450, 11000 Belgrade, Serbia; E-mail: \\ dragica@pharmacy.bg.ac.rs \\ ${ }^{4}$ Institute for Telecommunications and Electronics, IRITEL A.D. BELGRADE \\ Batajnički put 23, 11080 Belgrade, Serbia; E-mail:lebl@iritel.com
}

Abstract: In this paper we present the mathematical analysis of engagement of technicians in the teaching (educational) process on the faculty. The technicians receive the requests from professors and assistants. In the case that all technicians are busy in the moment of generating a new request, some of the assistants take over processing of the request. We analyse the probability of unwanted states, i.e. the states when it was necessary to engage the assistants on processing the request, which could have been processed by the technicians, if they had been free. The number of engaged technicians is determined in such a way that the probability of these unwanted states is satisfactorily small, i.e. that these states take available assistants' time in reasonable limits.

Keywords: queueing systems; teaching process; unwanted states

\section{Introduction}

Modern technology progress affects all aspects of human life. Among others, this progress is also visible in the faculty teaching (educational) process, where the efforts are made to implement modern methods of students' learning and testing [1]. Besides this, the great attention is devoted to the quality of faculty staff [2] and, indirectly, to the teaching quality. The quality of teaching process is not based only on the quality of teaching staff (professors and assistants), but also on the quality of the technicians support. 
Practical preparation and realization of the teaching process on many faculties is connected with engagement of technicians, who achieve contacts with students and prepare the necessary material for the teaching process. Technicians receive requests from professors, but also from assistants. Their engagement represents random process from the aspect of the requests, which they receive from professors and assistants, and from the aspect of necessary engagement time for each request. There are situations when all available technicians are engaged and the new request, which is generated in that moment, could not be processed (serviced). In such a situation it is possible that some assistant assumes execution of the request, which would have been done by the technician, if some technician had been free. This situation is not desirable, because it occupies the available time of the assistant, instead of using this time for assistant's main activity. But, if it doesn't happen too often, it can be reasonable and may be allowed to complete the necessary requests in time, instead of engaging new technicians. The analysis of the need for employing new technicians depends on the acceptable probability level that the assistants are engaged on processing (servicing) the requests.

In this paper we present mathematical model of teaching realization on the faculty. In the practice professors and assistants generate requests, which technicians process, and in the case that all technicians are busy, assistants take over the execution of these requests.

\section{Theoretical Base}

Queueing systems are often analyzed in literature [3], [4]. There are many contributions considering these systems. One interesting survey of different queueing systems is presented in [5]. The procedure of system defining in one specific case is presented in [6].

Queueing systems are described and analyzed using system states, which can be defined by the number of requests in the system, number of users, who generate requests in system, or the number of processing channels. The system, defined in one of these three ways, can be, for example, in some state $i$, which is designated as $\{i\}$.

Let us consider the part of one system, presented in Figure 1, which consists of three states. In some moment $t$ the system is in state $\{i-1\}$, or in state $\{i\}$, or in state $\{i+1\}$. Let us consider only the events connected with the state $\{i\}$. During the time $\Delta t$ (i.e. during the time interval $t-(t+\Delta t)$ ) the following events are possible:

- system is in moment $t$ in state $\{i-1\}$; during the time interval $\Delta t$ system passes into state $\{i\}$; the probability of this event can be expressed as $\lambda_{i-1} \cdot \Delta t$, where $\lambda_{i-1}$ is the arrival rate of new requests (intensity of new requests generation) in state $\{i-1\}$; 
- system is in moment $t$ in state $\{i\}$; during the time interval $\Delta t$ system passes into state $\{i+1\}$; the probability of this event can be expressed as $\lambda_{i} \cdot \Delta t$;

- in the moment $t$ system is in state $\{i\}$ and during the time interval $\Delta t$ passes into the state $\{i-1\}$; the probability of this event is $\mu_{i} \Delta t$, where $\mu_{i}$ is the service rate (intensity of processing the requests) in state $\{i\}$;

- in the moment $t$ system was in state $\{i\}$, and after time interval $\Delta t$ remains in the same state; the probability of this event is $1-\lambda \cdot \Delta t-\mu_{i} \cdot \Delta t$;

- in the moment $t$ system is in state $\{i+1\}$ and during the time interval $\Delta t$ passes into the state $\{i\}$; the probability of this event is $\mu_{i+1} \cdot \Delta t$.

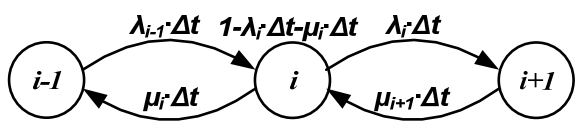

Figure 1

Diagram of possible states and possible transitions in the system

Let $P_{i-1}(t), P_{i}(t)$ and $P_{i+1}(t)$ present the probability that the system is in state $\{i-1\}$, $\{i\}$ and $\{i+1\}$, respectively. The probability that the system is in moment $t+\Delta t$ in the state $\{i\}$ can be expressed as:

$P_{i}(t+\Delta t)=P_{i}(t) \cdot\left(1-\lambda_{i} \cdot \Delta t-\mu_{i} \cdot \Delta t\right)+P_{i-1}(t) \cdot \lambda_{i-1} \cdot \Delta t+P_{i+1}(t) \cdot \mu_{i+1} \cdot \Delta t$

In the limiting case, when $\Delta t \rightarrow 0$, we obtain the differential equation:

$\frac{d P_{i}(t)}{d t}=-P_{i}(t) \cdot\left(\lambda_{i}+\mu_{i}\right)+P_{i-1}(t) \cdot \lambda_{i-1}+P_{i+1}(t) \cdot \mu_{i+1}$

The equations of this kind can be written for all possible states $\{i\}$ of the system, or in a matrix form:

$\frac{d \mathbf{P}(t)}{d t}=\mathbf{P}(t) \cdot \mathbf{A}$

where $\mathbf{P}(t)$ is the vector of state probabilities, and $\mathbf{A}$ is the matrix of transition intensities between the system states. In matrix $\mathbf{A}$ the elements on the diagonal represent the intensities of ,outgoing“ from the corresponding state, and are taken with the sign ,-“. The other elements in the matrix represent the intensities of ,incoming“ to the corresponding state, and are taken with the sign ,+"“.

After the sufficiently long period of time (i.e. when $t \rightarrow \infty$ ), the probabilities of system states tend to stationary values, i.e.:

$\frac{d \mathbf{P}(t)}{d t}=0$ 
According to (3) and (4), the system of differential equations becomes the system of linear equations:

$\mathbf{0}=\mathbf{P} \cdot \mathbf{A}$

The additional equation, which is necessary to solve this system of linear equations, is:

$\sum P_{i}=1$

where $\sum P_{i}$ includes probabilities of all possible states of the analyzed system.

\section{Analytical Model}

Let us analyse, in general case, one system, presented in Figure 2. The main parameters of this system are:

- $\quad k$ - number of professors, who generate requests in the system;

- $\quad m$ - number of assistants, who generate requests and, if necessary, process generated request;

- $\quad n$ - number of technicians, who process generated requests;

- $\quad \lambda$ - mean arrival rate expressed as number of arrivals in one second $\left(s^{-1}\right)$, i.e. mean intensity of requests generation by each professor and each assistant;

- $\quad \mu_{a}-$ mean assistants' service rate in $s^{-1}$, i.e. mean intensity of requests processing by each assistant;

- $\quad \mu_{t}-$ mean technicians' service rate in $s^{-1}$, i.e. mean intensity of requests processing by each technician.

In this system $\{i, j\}$ presents the state where requests are processed by $i$ assistants and $j$ technicians (for example, the state $\{1,2\}$ is the state where requests are processed by 1 assistant and 2 technicians).

Let us now analyse only one state $\{i, j\}$ (again $\{1,2\}$ ), Figure 2 . In this state the new request may be generated by $k$ professors and $m-1$ assistants. When this new request is generated (with total intensity $(k+m-1) \cdot \lambda$ ), the system passes to the state $\{1,3\}$.

We can come to state $\{1,2\}$ from the state $\{1,1\}$ in such a way that, also, $k$ professors and $m-1$ assistants generate new request with the intensity $(k+m-1) \cdot \lambda$.

The state $\{1,2\}$ is left when the processing of some request is finished. If an assistant finishes the processing, the system passes to the state $\{0,2\}$ (the intensity 
of this event is $\mu_{a}$ ). If one of two active technicians finishes the processing, the system passes to the state $\{1,1\}$ (the intensity of this event is $2 \cdot \mu_{t}$ ).

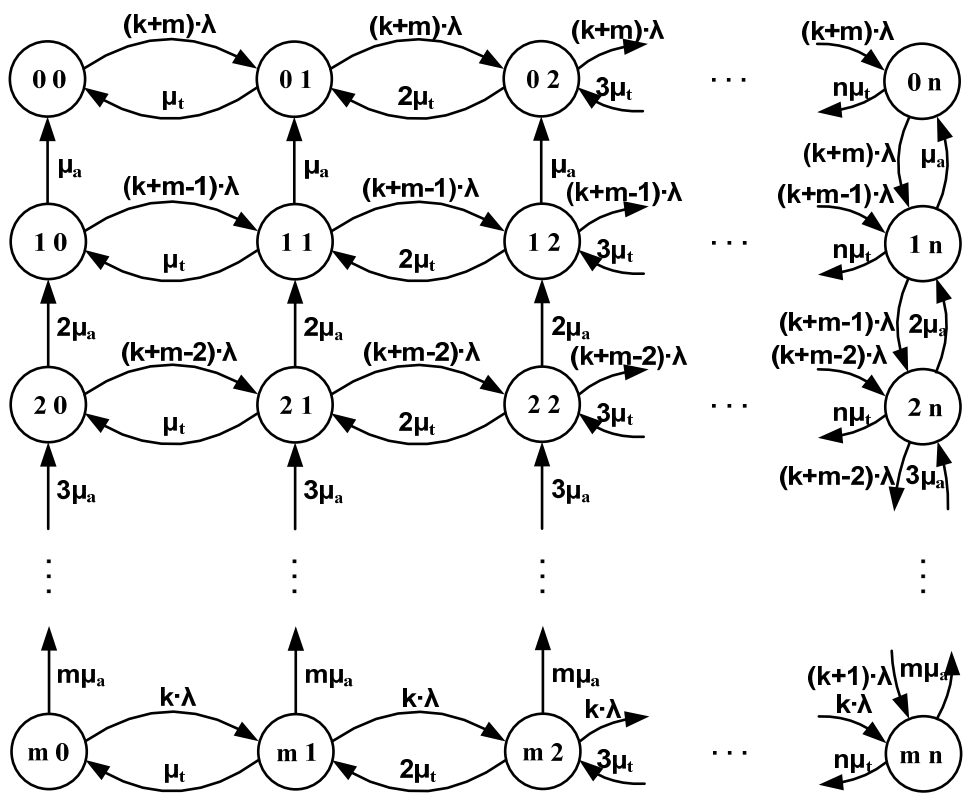

Figure 2

The model of teaching process on the faculty with $k$ professors, $m$ assistants and $n$ technicians

We can come to the state $\{1,2\}$ from the state $\{1,3\}$ in such a way that one of three active technicians finishes processing of the request (this happens with the intensity $\left.3 \cdot \mu_{t}\right)$, or from the state $\{2,2\}$ in such a way that one of two assistants, who processes the request, finishes the processing of his request (this happens with the intensity $2 \cdot \mu_{a}$ ).

The desirable states in the system are the ones, when assistants are not engaged on the processing of the requests, but the technicians succeed to process all requests, and the assistants have time for more important jobs. The result of these jobs is, among others, the generation of new requests. The undesirable states are the ones, when the assistants are engaged on the processing of the requests. The especially undesirable states are the ones, when the assistants are engaged on processing the requests, although one or more technicians are not engaged. The system can come to these states when we do not use the principle that free technicians take over the processing of the requests, which assistants started to process.

Let $p_{i j}$ presents the probability of state $\{i, j\}$, i.e. the probability of the state in which $i$ assistants and $j$ technicians are processing the requests. The probability of undesirable states (i.e. states when assistants are engaged on the processing of requests) can be calculated according to the formula: 
$p_{u}=1-\sum_{j=0}^{n} p_{0 j}$

The probability of especially undesirable states (i.e. states when assistants are engaged on the processing of requests, although there are free technicians) can be calculated using the formula:

$p_{u f t}=1-\sum_{j=0}^{n} p_{0 j}-\sum_{i=1}^{m} p_{i n}$

Table 1 presents the matrix of the transition intensities between the states of the system from Figure 2. When considering one transition, the initial state is read in the first column of the Table 1, and the final state is read in the first row of the Table 1.

\section{Results of the Analysis}

On the basis of the theoretical presentation from previous section, we shall analyse the system with the following characteristics:

- $\quad$ there are two professors in the system and they generate the requests;

- there is one technician in the system, who is engaged on processing the requests;

- $\quad$ there are 4 assistants in the system, and they can generate requests, and, if necessary, they can also process the requests;

- technician processes the requests with the mean intensity $\mu_{t}$;

- $\quad$ assistants process the requests with the mean intensity $\mu_{a}$;

- $\quad$ professors and assistants generate requests with the mean intensity $\lambda$;

- $\quad$ if some assistant is engaged on processing the request, he can not generate the requests;

- $\quad\{i, j\}$ is the state of the system when there are $i$ technicians and $j$ assistants, who are processing the requests;

- technician is the first to process the requests;

- $\quad$ if the technician is already occupied processing the request, assistants take over processing the new request. 
Table 1

Matrix of the transition intensities between the states of the system with $n$ technicians

\begin{tabular}{|c|c|c|c|c|c|c|c|c|c|c|c|c|c|c|c|c|c|}
\hline & 8 & $\sigma$ & $\vdots$ & ह & 으 & $\equiv$ & $\vdots$ & $\Xi$ & 8 & $\bar{c}$ & $\vdots$ & तี & $\vdots$ & ఏ & $\Xi$ & $\vdots$ & $\overline{\mathrm{E}}$ \\
\hline 8 & 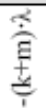 & 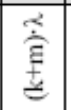 & $\vdots$ & 0 & 0 & 0 & $\vdots$ & 0 & 0 & 0 & $\vdots$ & 0 & $\vdots$ & 0 & 0 & $\vdots$ & 0 \\
\hline$\overline{0}$ & $\Xi$ & 全 & $\vdots$ & $\circ$ & 0 & 0 & $\vdots$ & 0 & 0 & 0 & $\vdots$ & 0 & $\vdots$ & 0 & 0 & $\vdots$ & $\circ$ \\
\hline 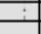 & i & i & $i$ & : & 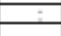 & 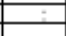 & 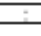 & 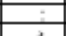 & i & 4 & 4 & 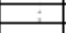 & 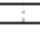 & 4 & 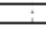 & 4 & 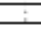 \\
\hline 5 & 0 & 0 & $\vdots$ & 产 & 0 & 0 & $\vdots$ & 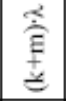 & 0 & 0 & $\vdots$ & 0 & $\vdots$ & 0 & 0 & $\vdots$ & 0 \\
\hline 으 & $\tilde{I}$ & 0 & $\vdots$ & 0 & 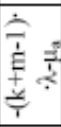 & 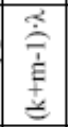 & $\vdots$ & 0 & 0 & 0 & $\vdots$ & 0 & $\vdots$ & 0 & 0 & $\vdots$ & 0 \\
\hline$=$ & 0 & \pm & $\vdots$ & 0 & $\Xi$ & 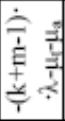 & $\vdots$ & 0 & 0 & 0 & $\vdots$ & 0 & $\vdots$ & 0 & 0 & $\vdots$ & 0 \\
\hline 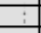 & : & $\div$ & $i$ & : & $\div$ & $\div$ & 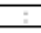 & $\because$ & i & : & $i$ & : & 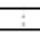 & : & : & : & : \\
\hline$\Xi$ & 0 & 0 & $\vdots$ & $\exists$ & 0 & 0 & $\vdots$ & 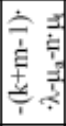 & 0 & 0 & $\vdots$ & 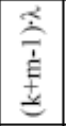 & $\vdots$ & 0 & 0 & $\vdots$ & 0 \\
\hline 이 & 0 & 0 & $\vdots$ & 0 & $\stackrel{\vec{H}}{\mathrm{~N}}$ & 0 & $\vdots$ & 0 & 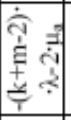 & 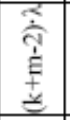 & $\vdots$ & 0 & $\vdots$ & 0 & 0 & $\vdots$ & 0 \\
\hline $\bar{N}$ & 0 & 0 & & 0 & 0 & $\stackrel{\vec{n}}{\mathrm{~d}}$ & & 0 & $\Xi$. & 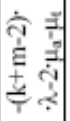 & & 0 & $\vdots$ & 0 & 0 & $\vdots$ & 0 \\
\hline 4 & i & $i$ & $i$ & i & 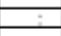 & 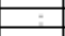 & 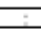 & 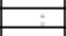 & i & i & $i$ & - & $i$ & $i$ & 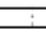 & 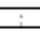 & 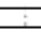 \\
\hline สี & 0 & 0 & $\vdots$ & 0 & 0 & 0 & $\vdots$ & $\stackrel{\vec{d}}{\mathrm{~d}}$ & 0 & 0 & $\vdots$ & 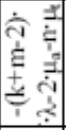 & $\vdots$ & 0 & 0 & $\vdots$ & $\vdots$ \\
\hline 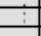 & $\div$ & $\div$ & $\div$ & $\div$ & 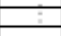 & 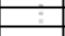 & 5 & 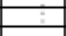 & $\div$ & $\square$ & $\div$ & : & $\div$ & $\div$ & $\div$ & $\div$ & 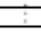 \\
\hline 으 & 0 & 0 & $\vdots$ & 0 & 0 & 0 & $\vdots$ & 0 & 0 & 0 & $\vdots$ & 0 & $\vdots$ & $\underset{\frac{3}{\dot{*}}}{\stackrel{3}{*}}$ & $\tilde{z}$ & $\vdots$ & 0 \\
\hline $\bar{\Xi}$ & 0 & 0 & $\vdots$ & 0 & 0 & 0 & $\vdots$ & 0 & 0 & 0 & $\vdots$ & 0 & $\vdots$ & $\Xi$ & 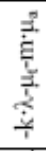 & $\vdots$ & $\circ$ \\
\hline 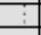 & : & $\vdots$ & $\vdots$ & 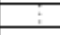 & 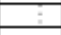 & 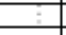 & 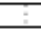 & 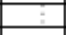 & $\bar{\vdots}$ & 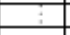 & 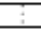 & $\dot{\vdots}$ & $\vdots$ & $7 \vdots$ & : & 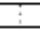 & $\vdots$ \\
\hline छ & 0 & 0 & $\vdots$ & 0 & 0 & 0 & $\vdots$ & 0 & 0 & 0 & $\vdots$ & 0 & $\vdots$ & 0 & 0 & $\vdots$ & 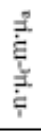 \\
\hline
\end{tabular}

Figure 3 presents the model, which illustrates the example where one technician processes the requests, and the matrix of the transition probabilities in that case is presented in Table 2. 


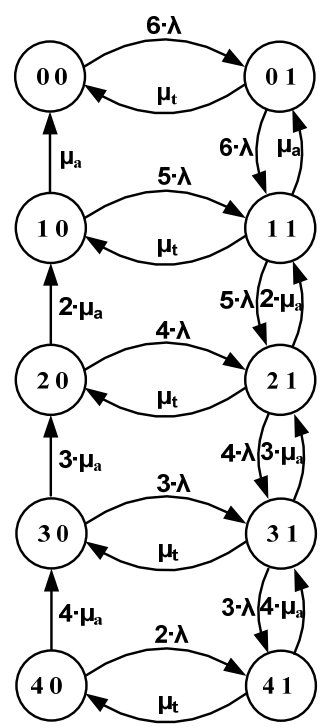

Figure 3

The model of the analyzed system with two professors, four assistants and one technician

Table 2

Matrix of the transition intensities between the states of the system with one technician

\begin{tabular}{|c|c|c|c|c|c|c|c|c|c|c|}
\hline & 00 & 01 & 10 & 11 & 20 & 21 & 30 & 31 & 40 & 41 \\
\hline 00 & $-6 \cdot \lambda$ & $\mu_{\mathrm{t}}$ & $\mu_{\mathrm{a}}$ & 0 & 0 & 0 & 0 & 0 & 0 & 0 \\
\hline 01 & $6 \cdot \lambda$ & $-6 \cdot \lambda-\mu_{\mathrm{t}}$ & 0 & $\mu_{\mathrm{a}}$ & 0 & 0 & 0 & 0 & 0 & 0 \\
\hline 10 & 0 & 0 & $-5 \cdot \lambda-\mu_{\mathrm{a}}$ & $\mu_{\mathrm{t}}$ & $2 \cdot \mu_{\mathrm{a}}$ & 0 & 0 & 0 & 0 & 0 \\
\hline 11 & 0 & $6 \cdot \lambda$ & $5 \cdot \lambda$ & $\begin{array}{c}-5 \cdot \lambda-\mu_{\mathrm{t}^{-}} \\
\mu_{\mathrm{a}}\end{array}$ & 0 & $2 \cdot \mu_{\mathrm{a}}$ & 0 & 0 & 0 & 0 \\
\hline 20 & 0 & 0 & 0 & 0 & $-4 \cdot \lambda-2 \cdot \mu_{\mathrm{a}}$ & $\mu_{\mathrm{t}}$ & $3 \cdot \mu_{\mathrm{a}}$ & 0 & 0 & 0 \\
\hline 21 & 0 & 0 & 0 & $5 \cdot \lambda$ & $4 \cdot \lambda$ & $-4 \cdot \lambda-\mu_{\mathrm{t}}-2 \cdot \mu_{\mathrm{a}}$ & 0 & $3 \cdot \mu_{\mathrm{a}}$ & 0 & 0 \\
\hline 30 & 0 & 0 & 0 & 0 & 0 & 0 & $-3 \cdot \lambda-3 \cdot \mu_{\mathrm{a}}$ & $\mu_{\mathrm{t}}$ & $4 \cdot \mu_{\mathrm{a}}$ & 0 \\
\hline 31 & 0 & 0 & 0 & 0 & 0 & $4 \cdot \lambda$ & $3 \cdot \lambda$ & $-3 \cdot \lambda-3 \cdot \mu_{\mathrm{a}}-\mu_{\mathrm{t}}$ & 0 & $4 \cdot \mu_{\mathrm{a}}$ \\
\hline 40 & 0 & 0 & 0 & 0 & 0 & 0 & 0 & 0 & $-2 \cdot \lambda-4 \cdot \mu_{\mathrm{a}}$ & $\mu_{\mathrm{t}}$ \\
\hline 41 & 0 & 0 & 0 & 0 & 0 & 0 & 0 & $3 \cdot \lambda$ & $2 \cdot \lambda$ & $-4 \cdot \mu_{\mathrm{a}}-\mu_{\mathrm{t}}$ \\
\hline
\end{tabular}

According to Figure 3, the desirable states in this system are $\{0,0\}$ and $\{0,1\}$. All other states are undesirable, and the probability of this situation $\left(p_{u}\right)$ can be calculated using the formula (7).

Figure 4 presents the probabilities $p_{u}$ for the analyzed system with one technician. The probabilities of these states are read on $y$ axis, and the intensity $\mu_{t}$ is read on the $x$ axis. The parameter for the presented curves is intensity $\mu_{a}\left(\mu_{a}\right.$ ranges from 1 to 5). The curves are presented for two values of intensities of requests generation: $\lambda=0.1$ and $\lambda=0.5$. 


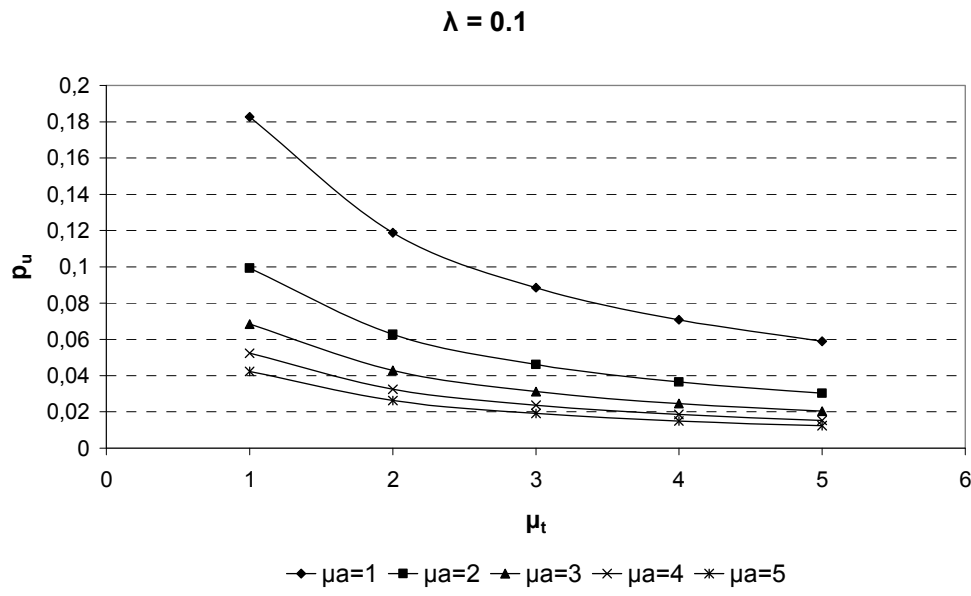

$\lambda=0.5$

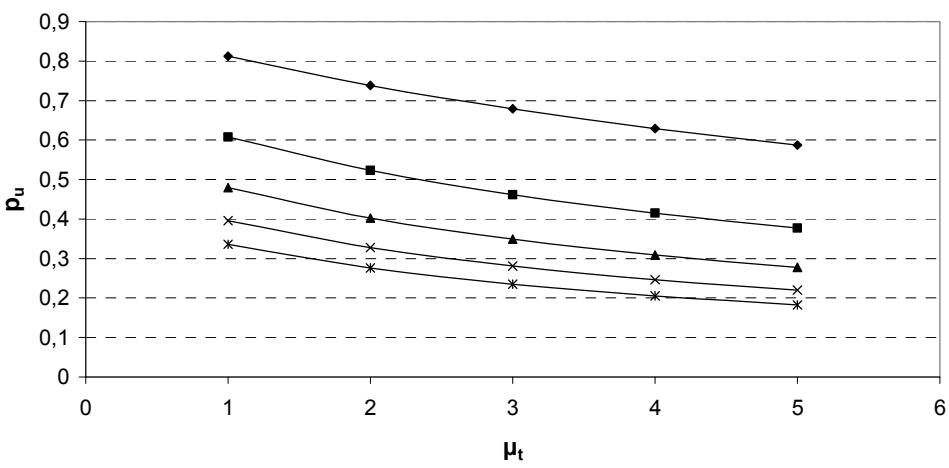

$-\mu \mathrm{a}=1-\mu \mathrm{a}=2 \rightarrow \mu \mathrm{a}=3 * \mu \mathrm{a}=4 \rightarrow \mu \mathrm{a}=5$

Figure 4

Probabilities of undesirable states in the system with one technician, in which assistants must be engaged on processing the requests

In the system, which is here analyzed, the states $\{1,0\},\{2,0\},\{3,0\}$ and $\{4,0\}$ are especially undesirable. The probability of such a state $\left(p_{u f f}\right)$, in which the assistants are engaged on the processing of the requests, and the technician is free, can be calculated using the formula (8).

Figure 5 presents the probability of especially undesirable state $p_{u f t}$ in the system with one technician. 


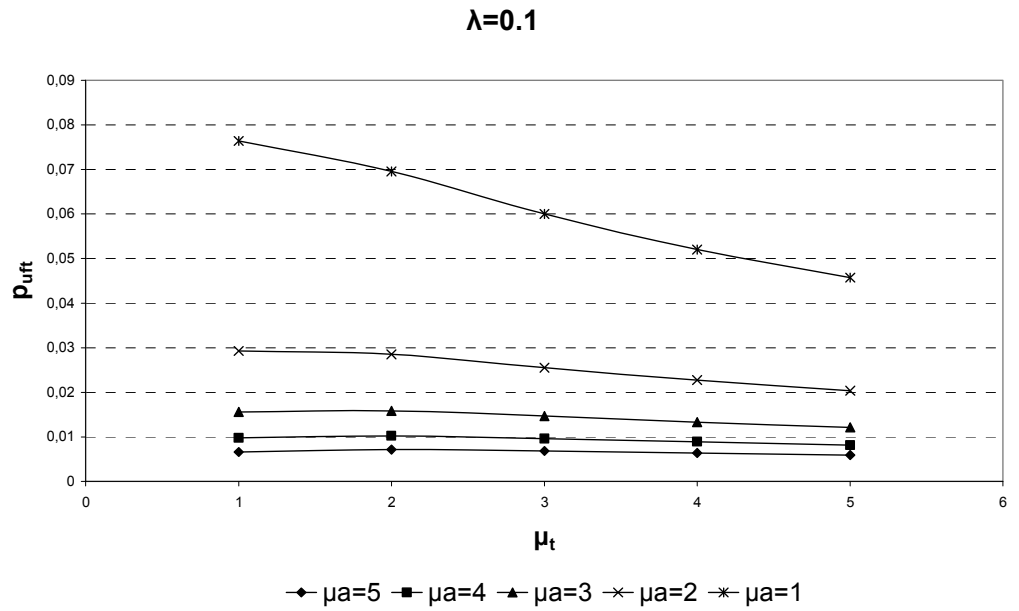

$\lambda=0.5$

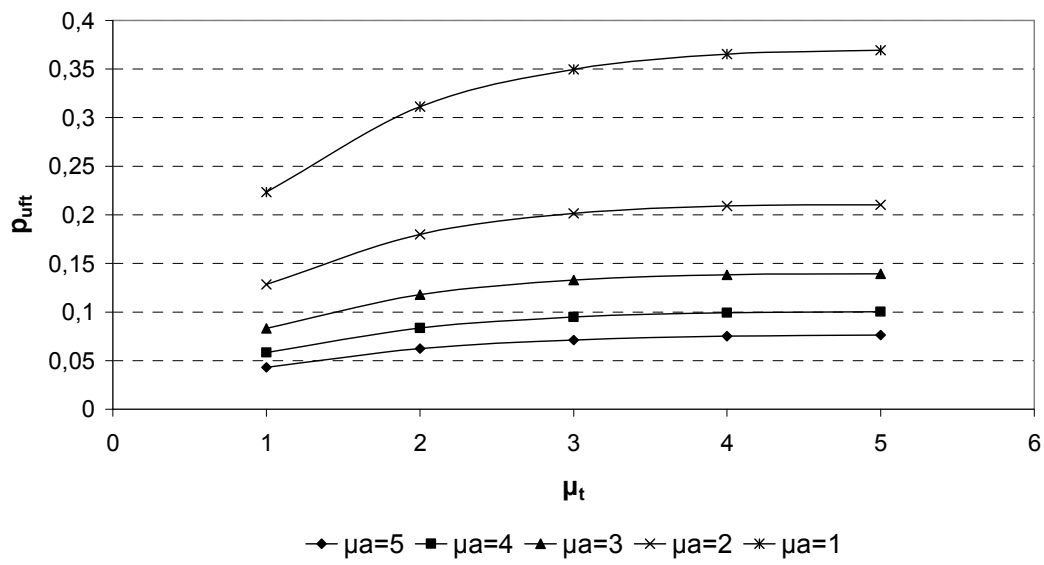

Figure 5

Probabilities of especially undesirable states in the system with one technician

We shall compare the presented results for the system with one technician to the results for the system of the same type, with the only difference that two technicians are engaged on the processing of the requests. 


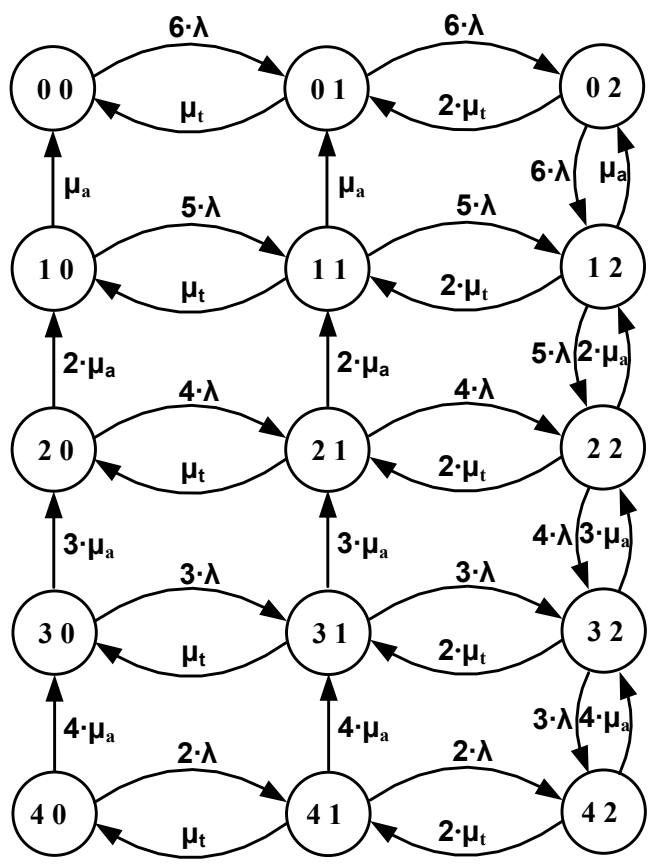

Figure 6

The model of the analyzed system with two professors, four assistants and two technicians

Figure 6 presents the model, which illustrates the analyzed example, where two technicians process the requests, and the matrix of transition probabilities in that case is presented in Table 3.

According to Figure 6, in the system, which is analyzed, the desirable states are $\{0,0\},\{0,1\}$ and $\{0,2\}$. All other states are undesirable, and the probability of such a situation $\left(p_{u}\right)$ is calculated, again, using the formula (7).

Figure 7 presents the probabilities $p_{u}$ for the analyzed system with two technicians.

The probability of especially undesirable state $\left(p_{u f t}\right)$, in which the assistants are engaged on the processing of requests although one or both technicians are free, is calculated using formula (8).

Figure 8 presents the probability $p_{u f t}$ in the system with two technicians. 
Table 3

Matrix of the transition intensities between the states of the system with two technicians

\begin{tabular}{|c|c|c|c|c|c|c|c|c|c|c|c|c|c|c|c|}
\hline & 8 & $\overrightarrow{0}$ & $\delta$ & 은 & $=$ & I & ㅇ & $\bar{N}$ & ป & లి & $\bar{m}$ & થี & ㅇ & $\bar{J}$ & F \\
\hline 8 & $\widetilde{\phi}$ & $\Xi$ & 0 & $=$ & 0 & 0 & 0 & 0 & 0 & 0 & 0 & 0 & 0 & 0 & 0 \\
\hline$\overline{0}$ & $\tilde{b}$ & $\frac{\overrightarrow{1}}{\frac{1}{\dot{1}}}$ & $\underset{\dot{\sim}}{\vec{v}}$ & 0 & $=$ & 0 & 0 & 0 & 0 & 0 & 0 & 0 & 0 & 0 & 0 \\
\hline$\widehat{\delta}$ & 0 & $\widehat{\dot{b}}$ & 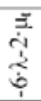 & 0 & 0 & $=$ & 0 & 0 & 0 & 0 & 0 & 0 & 0 & 0 & 0 \\
\hline$\underline{-}$ & 0 & 0 & 0 & 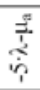 & $\Xi$ & 0 & 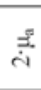 & 0 & 0 & 0 & 0 & 0 & 0 & 0 & 0 \\
\hline$=$ & 0 & 0 & 0 & $\vec{r}$ & 㫄 & $\underset{\dot{\sim}}{\vec{i}}$ & 0 & 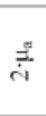 & 0 & 0 & 0 & 0 & 0 & 0 & 0 \\
\hline$\simeq$ & 0 & 0 & $\hat{\xi}$ & 0 & $\dot{\dot{n}}$ & 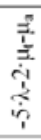 & 0 & 0 & $\stackrel{\vec{n}}{\stackrel{\sim}{\sim}}$ & 0 & 0 & 0 & 0 & 0 & 0 \\
\hline હે & 0 & 0 & 0 & 0 & 0 & 0 & 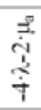 & $\Xi$ & 0 & 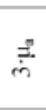 & 0 & 0 & 0 & 0 & 0 \\
\hline $\bar{\sim}$ & 0 & 0 & 0 & 0 & 0 & 0 & $\stackrel{\leftrightarrow}{\dot{q}}$ & 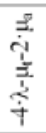 & $\stackrel{\bar{\sim}}{\dot{\sim}}$ & 0 & $\stackrel{\mathscr{r}}{\dot{m}}$ & 0 & 0 & 0 & 0 \\
\hline ป & 0 & 0 & 0 & 0 & 0 & is & 0 & $\stackrel{\leftrightarrow}{\dot{q}}$ & 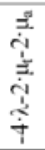 & 0 & 0 & $\stackrel{乛}{\dot{m}}$ & 0 & 0 & 0 \\
\hline 료 & 0 & 0 & 0 & 0 & 0 & 0 & 0 & 0 & 0 & & $\Xi$ & 0 & $\stackrel{\mathscr{H}}{\dot{+}}$ & 0 & 0 \\
\hline $\bar{m}$ & 0 & 0 & 0 & 0 & 0 & 0 & 0 & 0 & 0 & $\widetilde{i}$ & 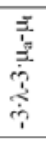 & $\stackrel{\vec{\sim}}{\vec{\sim}}$ & 0 & 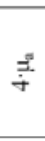 & 0 \\
\hline ชี & 0 & 0 & 0 & 0 & 0 & 0 & 0 & 0 & $\stackrel{\leftrightarrow}{\dot{\tau}}$ & 0 & $\stackrel{2}{n}$ & 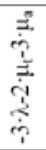 & 0 & 0 & $\stackrel{f}{\dot{H}}$ \\
\hline P & 0 & 0 & 0 & 0 & 0 & 0 & 0 & 0 & 0 & 0 & 0 & 0 & 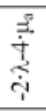 & $\Xi$ & 0 \\
\hline $\bar{\exists}$ & 0 & 0 & 0 & 0 & 0 & 0 & 0 & 0 & 0 & 0 & 0 & 0 & $\stackrel{\leftrightarrow}{\sim}$ & 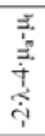 & $\underset{\dot{i}}{\vec{i}}$ \\
\hline$\stackrel{Y}{F}$ & 0 & 0 & 0 & 0 & 0 & 0 & 0 & 0 & 0 & 0 & 0 & $\stackrel{\leftrightarrow}{r}$ & 0 & $\stackrel{\leftrightarrow}{\dot{\sim}}$ & 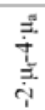 \\
\hline
\end{tabular}


$\lambda=0.1$

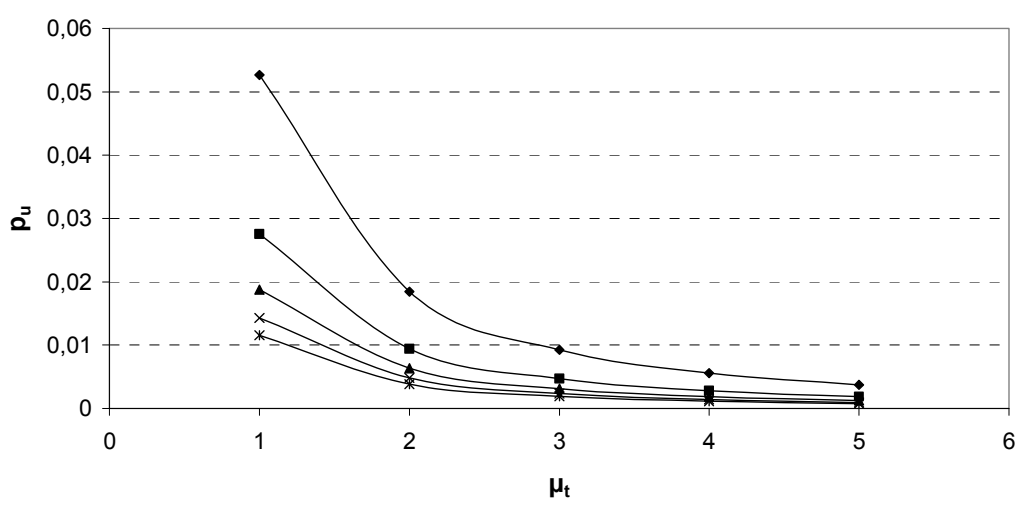

$\rightarrow \mu \mathrm{a}=1-\mu \mathrm{a}=2 \rightarrow \mu \mathrm{a}=3 * \mu \mathrm{a}=4-\mu \mathrm{a}=5$

$\lambda=0.5$

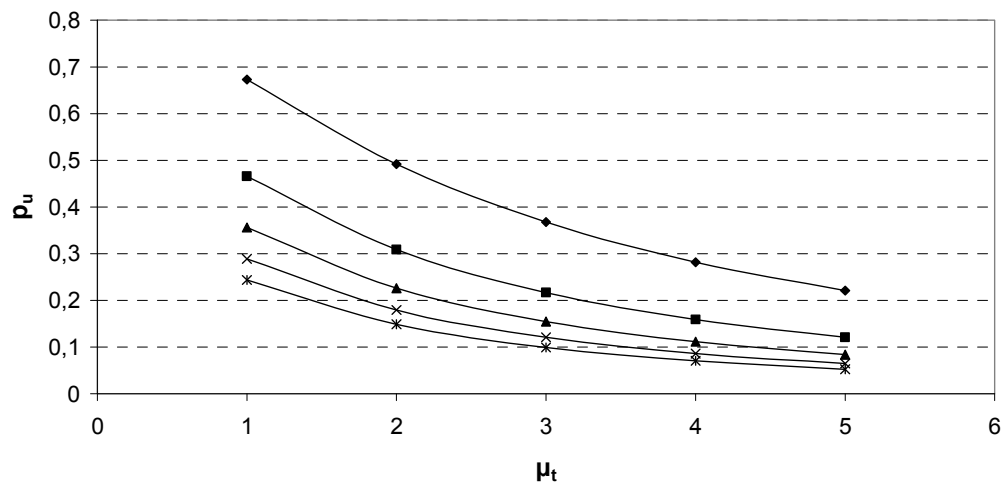

$\rightarrow \mu \mathrm{a}=1 \rightarrow-\mu \mathrm{a}=2 \rightarrow \mu \mathrm{a}=3 \rightarrow \mu \mathrm{a}=4 \rightarrow-\mu \mathrm{a}=5$

Figure 7

The probability of undesirable states in the system with two technicians, in which assistants must be engaged on processing the requests 

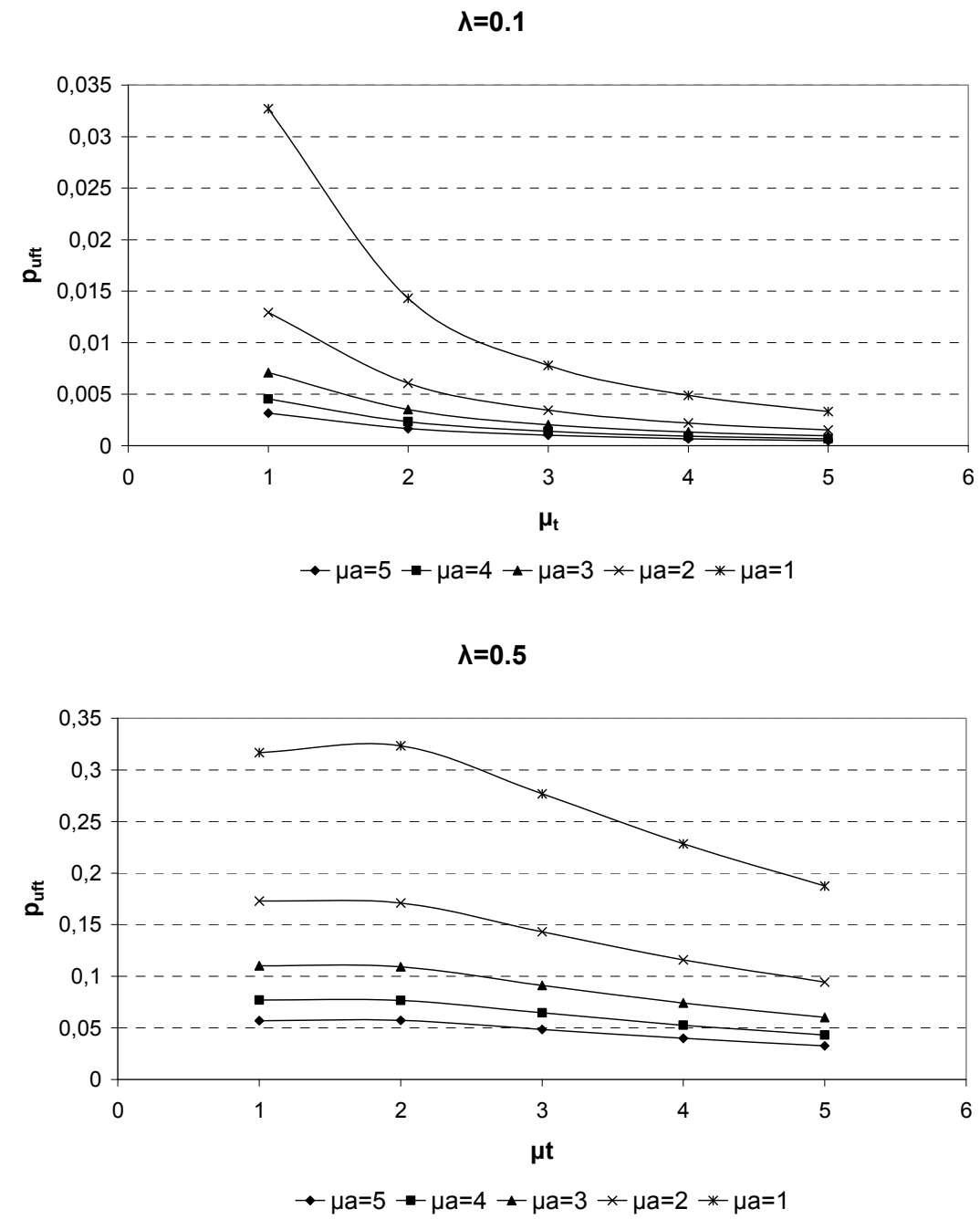

Figure 8

The probabilities of especially undesirable states in the system with two technicians 


\section{Analysis of the Calculated Results}

Comparing the probabilities of assistants' engagement in the cases when there is one and when there are two technicians in the system, we can say that it is possible to reduce the probability of assistants' engagement more than twice when there are two technicians in the system instead of one technician, although the intensity of processing the requests is the same in both cases. For example, if $\lambda=0.1, \mu_{t}=1, \mu_{a}=1$, the probability of assistants' engagement is 0.18 when there is one technician in the system (Figure 4), and 0.052 when there are two technicians in the system (Figure 7). This is much better than in the case when the technician processes the requests two times faster $\left(\mu_{t}=2\right)$, and the assistants continue to process the requests with the same intensity $\left(\mu_{a}=1\right)$, because in that case the probability of assistants' engagement on processing requests is 0.12 (Figure 4).

Similar results are obtained comparing the probabilities of especially undesirable states, in which assistants are engaged on the processing of requests, although there are free technicians. For example, if $\lambda=0.1, \mu_{t}=1, \mu_{a}=1$ the probability of these, especially undesirable states is 0.077 when there is one technician in the system (Figure 5), and 0.033 when there are two technicians in the system (Figure $8)$. This is much better than in the case when the technician processes the requests two times faster $\left(\mu_{t}=2\right)$, and the assistants continue to process the requests with the same intensity $\left(\mu_{a}=1\right)$, because in that case the probability of especially undesirable states is 0.07 (Figure 5).

If it is $\lambda=0.5$ and $\mu_{t}<3$ in the case of one technician, or $\mu_{t}<1.5$ in the case of two technicians, system functions in the state where the traffic of generated requests is greater than the traffic, which can be processed by the technicians, i.e.:

$$
\frac{(k+m) \cdot \lambda}{n \cdot \mu_{t}}>1
$$

The state, when the condition expressed by formula (9) is satisfied, is known in the theory as the state when the system is unstable.

The graphs of especially undesirable states in the case that $\lambda=0.5$ have, seemingly, illogical shape, because the graphs are growing functions (i.e., when the technicians process the request faster, the probability of especially undesirable states increases). If the system functions in these conditions, it is not dimensioned well, i.e. it is necessary to avoid system operation in the state where total intensity of requests generation is greater than total intensity of technicians' processing of the requests.

\section{Conclusion}

In this paper we present the method for determination of required number of technicians for the teaching process implementation on the faculty. The technicians are engaged on the processing of the professors' and assistants' 
requests. If in the moment of generating the new request there are no free technicians, processing of the request takes over one of the assistants. It is important that such an event is as rare as possible, because in that case assistant can't do his regular job. On the other hand, if there are too many technicians, they are not adequately engaged on their job, i.e. they will have free time. The required number of technicians is situated between these two extreme cases. This required number of technicians can be determined depending on the allowed level of assistants' engagement for processing the requests (which also could have been done by the technicians, if they had been free).

\section{References}

[1] Maravić Čisar, S., Radosav, D., Markoski, B., Pinter, R., Čisar, P.: Computer Adaptive Testing of Student Knowledge, in Acta Polytechnica Hungarica, Vol. 7, No. 4, 2010, pp. 139-152

[2] Stoklasa, J., Talašová, J., Hoček, P.: Academic Staff Performance Evaluation - Variants of Models, in Acta Polytechnica Hungarica, Vol. 8, No. 3, 2011, pp. 91-111

[3] Kleinrock, L.: Queueing Systems, John Wiley \& Sons, New York, 1975

[4] Teletraffic Engineering Handbook: ITU-D SG 2/16\&ITC, Draft 2001-0620

[5] Filipowitz, B., Kwiecien, J.: Queueing Systems and Networks. Models and Applications, Bulletin of the Polish Academy of Sciences, Technical Sciences, Vol. 56, No. 4, December 2008, pp. 379-390

[6] Petrović, G., Petrović, N., Marinković, Z.: Application of the Markov Theory to Queueing Networks, Facta Universitatis, Series Mechanical Engineering, Vol. 6, No. 1, 2008, pp. 45-56 